研

究

\title{
Finite Element Analysis of PIM Filling Process with Slip Characterization of Powder-Binder Mixtures
}

\author{
C. J. Hwang and T. H. Kwon
}

Department of Mechanical Engineering, Pohang University of Science and Technology, San 31 Hyoja Dong, Pohang 790-784, Korea.

Received June 4, 1999

\section{SYNOPSIS}

Powder Injection Molding (PIM) filling process is one of the key processes towards a successful PIM. In this regard, the methods of slip characterization of powder-binder mixtures and a quasi-3-dimensional finite element simulation program based on Hele-Shaw approximation has been presented.

In contrast to conventional injection molding of thermoplastics, there are many PIM parts that include thick regions. To simulate such thick PIM parts properly, a full 3-dimensional simulation program is needed. In this regard, we have been developing a full 3-dimensional finite element program for PIM filling process with the slip phenomena taken into account. For a computational efficiency, a pre-meshed finite element mesh was used with a scalar fillfactor to automatically update the computational domain during the filling.

\section{KEY WORDS}

Powder Injection Molding (PIM), Mold Filling, Slip Characterization, Finite Element Analysis, Powder-Binder Mixture

\section{Introduction}

Powder Injection Molding (PIM) is a new powder forming process that has been evolved from the injection molding process of polymers. It is well known that the injection molding is one of the key processes towards a successful PIM that is composed of several processes ${ }^{1}$. For PIM filling process the CAE system for the plastic injection molding process can be applied with some modifications in order to account for rheological characteristics of powderbinder mixtures. To date, the CAE system for the PIM filling analysis which was developed is capable of analyzing the flow only in thin mold cavity which has a large aspect ratio (length to thickness) ${ }^{2,3)}$. But one of the major advantages of PIM is a low production cost for complicated parts, especially for the parts with a small aspect ratio. In the conventional powder compaction forming process, only two-dimensional (profile) cavities can be made and parts with a high aspect ratio (length to thickness) can not be pressed in the length direction. Three-dimensional cavities can be formed easily using PIM, and the dimensional tolerance of the parts can be kept at a minimum level because of uniform shrinkage upon sintering and a relatively small wear level of the mold through a long production period. The ability to form the final geometry in the PIM process saves the secondary machining cost. So there are many PIM products which have complicated 3-dimensional geometries. For better understanding of PIM process, it is of importance to obtain accurate finite analysis program that can be applied to the full 3-dimensional geometries. In these regards, it is of our interest to develop a reliable full 3-dimensional CAE system for the PIM filling process.

It is nowadays well known that powder/binder mixtures have a peculiar rheological feature of the so-called apparent slip phenomena at the wall ${ }^{4}$. Therefore, a proper numerical simulation of the PIM filling process essentially requires a proper full 3-dimensional finite element formulation including the slip phenomena of powder-binder mixtures.

In the present paper, the methods of slip characterization of powder-binder mixtures and a quasi-3-dimensional finite element program based on Hele-Shaw approximation are presented. And a full 3-dimensional finite element formulation into a numerical simulation system including the slip phenomena for PIM filling process is also presented.

\section{Slip Characterization of Powder-Binder Mixtures}

It is essential to characterize the slip phenomena in obtaining rheological data for powder-binder mixtures. There are two possible methods of characterizing the apparent slip phenomena: slip velocity model and slip layer model. In contrast to Fig.1-(a), which represents a normal 
velocity profile for the non-slip boundary condition, the concepts of the slip velocity and the slip layer models are shown in Fig.1-(b) and (c), respectively. A proper rheological characterization of powder-binder mixtures should involve the determination not only for the viscosity as a function of the shear rate and temperature, as usual, but also of the slip velocity or the slip layer thickness as a function of the relevant dependent variables.

Mooney ${ }^{51}$ proposed a simple expression to determine the slip velocity $\left(V_{S}\right)$

$$
8 V_{s}=\left.\frac{\partial\left(32 Q / \pi D^{3}\right)}{\partial(1 / D)}\right|_{\tau_{w}}
$$

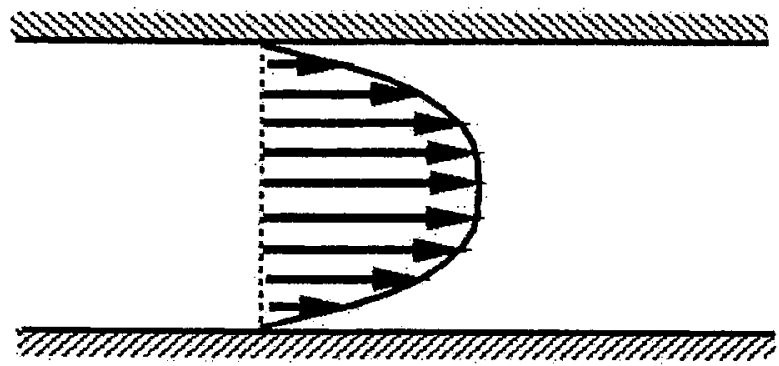

(a)

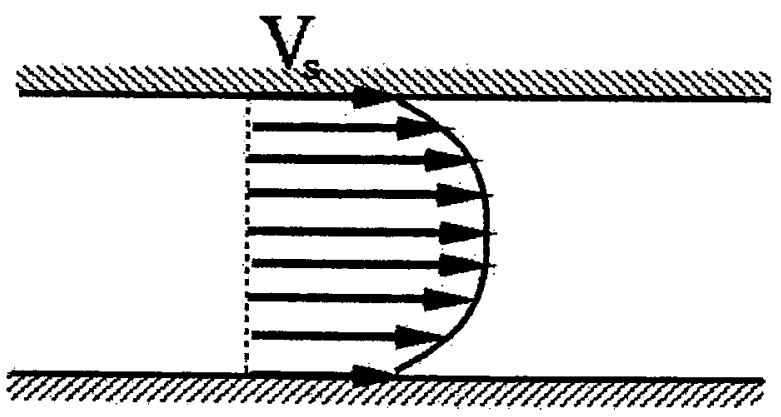

(b)

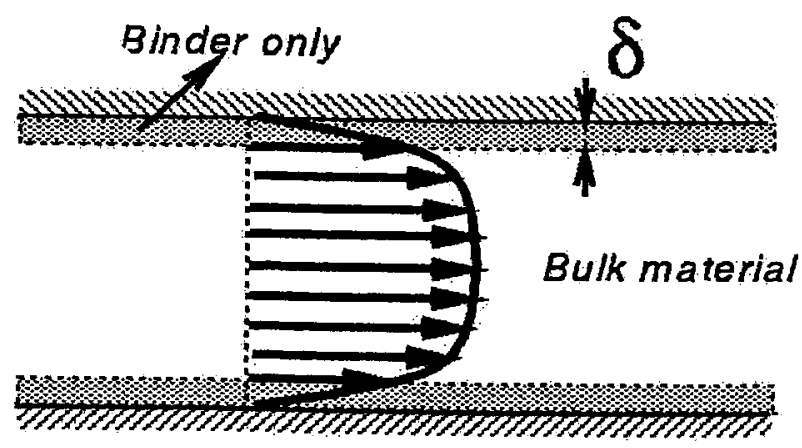

(c)

Fig.1 Schematic velocity profiles at mold wall and within powderbinder mixture for various models of PIM.
To make use of the above formula, one has to obtain the relationship between the flowrate $(Q)$ and wall shear stress $\left(\tau_{w}\right)$ from a capillary rheometer with several capillary dies of different diameter $(D)$.

Some attempts to determine the slip layer thickness have been reported ${ }^{4,6,7)}$. We proposed a new characterization method for slip layer thickness ${ }^{3)}$, which differs slightly from the previous methods. We expressed the slip layer thickness by the formula

$$
\delta=\left.\left(\frac{\partial\left(32 Q / \pi D^{3}\right)}{\partial(1 / D)} / 8 \dot{\gamma}_{2 w}-\dot{\gamma}_{1 w}\right)\right|_{\tau_{w}}
$$

where $\gamma_{i w}$ and $\gamma_{2 w}$ represent the wall shear rate of the binder material in the slip layer and that of the bulk mixture, respectively, when subjected to the wall shear stress.

It was found that both the slip velocity and the slip layer thickness could be curve fitted well as a power law function of the wall shear stress $\left(\tau_{w}\right)$ for various metal powder-binder PIM mixtures as well as for other particle filled materials. A typical plot of $V_{s}$ vs. $\tau_{w}$ is presented in Fig. 2 for mixture composed of a stainless steel powder and a EVA based binder system ${ }^{3)}$.

Once $V_{s}$ has been determined as a function of $\tau_{w}$, the bulk shear viscosity as a function of shear rate can be determined by the usual Raninowitsch correction, taking account of the true shear rate after the slip effect. The nonNewtonian viscosity obtained in this way was curve fitted in terms of the modified Cross model with a yield stress $\left(\tau_{y}\right)$ given by

$$
\eta(\dot{\gamma}, T)=\frac{\eta_{0}}{1+C\left(\eta_{\mathrm{o}} \dot{\gamma}\right)^{1-\mu}}+\frac{\tau_{y}}{\dot{\gamma}} \text { with } \eta_{0}=B \exp \left(\frac{T_{B}}{T}\right)
$$

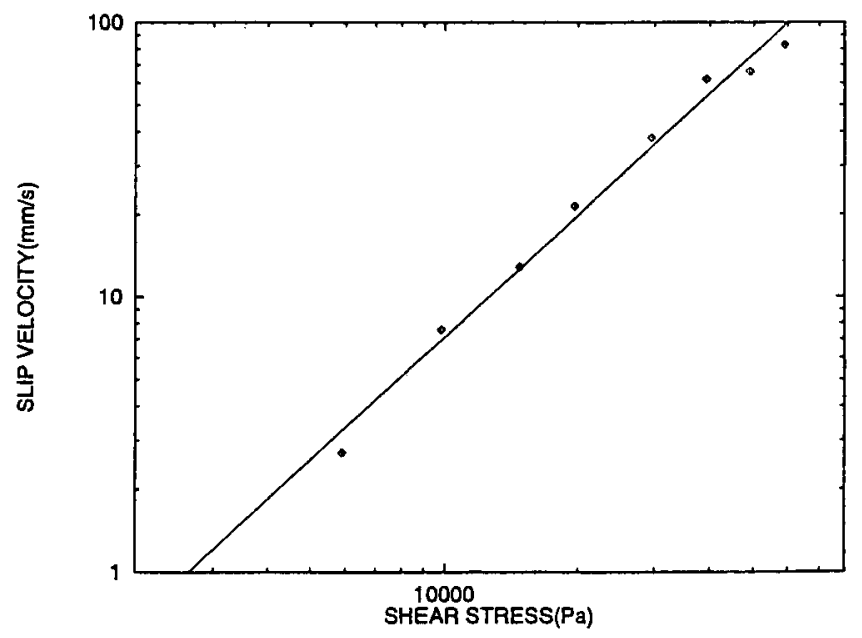

Fig.2 Slip velocity as a function of wall shear stress. 


\section{Quasi-3 Dimensional Finite Element Modeling for PIM Filling Process with Slip Effect}

A quasi-3 Dimensional finite element simulation program for the PIM filling process was developed based on the models discussed above, using a slip velocity model or a slip layer model to represent the apparent slip phenomena of the powder-binder mixtures. The program developed can be applied to thin walled parts.

In the slip velocity model, a slip velocity is applied at the solid wall as a boundary condition. In contrast, the slip layer model assumes that an extremely thin slip layer consisting only of the binder system exists next to the wall, while most of the flow region is occupied by the bulk powder-binder mixture, and that a non-slip condition applies at the wall.

In both slip models, the fundamental governing expressions representing the cavity filling process with a Hele-Shaw approximation are derived. The newly derived pressure equations for both slip models can be summarized as follows ${ }^{2}$ :

For the slip velocity model,

$$
\frac{\partial}{\partial x}\left(\tilde{s} \frac{\partial p}{\partial x}-b u_{s}\right)+\frac{\partial}{\partial y}\left(\tilde{s} \frac{\partial p}{\partial y}-b v_{s}\right)=0
$$

where $u_{s}$ and $v_{s}$ denoting slip velocity components and the fluidity constant is defined as follows:

$$
\bar{s} \int_{\varepsilon}^{b} \frac{z^{2}}{\eta_{1}} d z
$$

For the slip layer model,

$$
\frac{\partial}{\partial x}\left(\hat{S} \frac{\partial p}{\partial x}\right)+\frac{\partial}{\partial y}\left(\hat{S} \frac{\partial p}{\partial y}\right)=0
$$

with the fluidity constant being defined by as follows:

$$
\hat{S}=\int_{\varepsilon}^{\psi} \frac{z^{2}}{\eta_{1}} d z+\int_{\psi}^{b} \frac{z^{2}}{\eta_{2}} d z
$$

where $\eta_{1}, \eta_{2}$ denote viscosity of the bulk powder-binder mixture and the pure binder system in the slip layer, respectively. The lower bound of the first integral term $\varepsilon$ indicates the thickness of a region where no yield takes place while $\Psi$ in the upper bound represents the thickness of the bulk materials such that $(b-\Psi)$ is the slip layer thickness.

Once the pressure field is obtained from the solution of pressure equation, depending on the slip model chosen, one can find the local velocity distribution and can change accordingly the computational domain occupied by the material and repeat the finite element analysis until the whole cavity has been filled. Both pressure equations are non-linear partial differential equations, which require iterative methods to obtain a convergent numerical solution. A finite element method has been employed to solve them, whereas the energy equation for the temperature field is solved by a finite difference method. The detailed numerical method and the solution scheme are given in Ref.(2).

\section{Full 3-Dimensional Finite Element Modeling for PIM Filling Process with Slip Effect}

Due to high viscosity of polymeric materials, the viscous dissipation may cause the temperature to vary significantly over the flow domain. Since the viscosity depends on the temperature, energy equation has to be solved to obtain temperature field in addition to the momentum and continuity equations. In the present work, however, isothermal flow is assumed to focus our study on the simulation of the filling process with the slip effect taken into account. Consequently, in the present work, flow has been assumed to be isothermal, non-Newtonian and incompressible flow. And quasi-steady assumption is used during the mold filling process at each time.

The momentum and continuity equations governing the flow are:

where $\sigma$ is the stress tensor, $\mathbf{f}$ is the body force per unit volume, $\rho$ is the density of the fluid, $\mathbf{u}$ is the velocity and $\Omega$ is a bounded region (flow domain) in $R^{3}$.

$$
\begin{array}{cl}
\nabla \cdot \sigma+\mathbf{f}=\rho \frac{D \mathbf{u}}{D t} & \text { in } \Omega, \\
\nabla \cdot \mathbf{u}=0 & \text { in } \Omega,
\end{array}
$$

For a well posed problem, the boundary conditions to be satisfied are

$$
\begin{aligned}
& \sigma \cdot \mathbf{n}=\overline{\mathrm{T}} \text { on } \Gamma_{\mathrm{T}} \text {, } \\
& \mathbf{u}=\overline{\mathbf{u}} \text { on } \Gamma_{\mathbf{u}} \text {, }
\end{aligned}
$$

where $\mathbf{n}$ is the outward normal vector at the boundary, $\Gamma_{\mathrm{T}}$ is the part of the boundary where the traction vector is given, $\Gamma_{u}$ is the part of the boundary where the velocity is given, $\bar{T}$ is the prescribed value of traction vector on $\Gamma_{\mathrm{T}}$ and $\overline{\mathbf{u}}$ is the prescribed value of velocity on $\Gamma_{\mathrm{u}}$.

For highly filled suspensions, the slip may become macroscopically important. The slip velocity, that is, the difference between the tangential velocity of the wall and the velocity of the fluid layer adjacent to that wall, depends on the shear stress at the solid boundary, the temperature, etc. As a general concept, it is assumed that the slip velocity has the same direction as the shear component of the traction. Then, the form of the boundary conditions when 
slip occurs is ${ }^{8,9)}$ :

$$
\begin{aligned}
& \left(\mathbf{V}_{b}-\mathbf{u}\right) \cdot \mathbf{s}=V_{s} \\
& \left(\mathbf{V}_{b}-\mathrm{u}\right) \cdot \mathbf{n}=\mathbf{0}
\end{aligned} \text { on } \Gamma_{S}
$$

where $\Gamma_{S}$ is the part of the boundary where slip occurs, $s$ is the unit tangential vector on the wall surface, $\mathbf{n}$ is the outward unit normal vector on the wall surface, $V_{S}$ is the velocity at the wall and $V_{b}$ is the velocity of the wall.

$$
V_{s}=\alpha(T) \tau_{w}{ }^{n}, \alpha(T)=a \exp (b T),
$$

For simplicity, it is assumed that, based on our experimental data, the slip velocity is a function of the wall shear stress and temperature ("slip law") as follows" where $a, b, m$ are material constants.

With the help of the slip law and new type of mixed boundary condition and penalty parameter, the components of the traction vectors can be represented as follows:

$$
\begin{aligned}
& t_{l}=\frac{\left|\mathbf{V}_{b}-\mathbf{u}\right|^{1 / m}}{\alpha^{1 / m}} \frac{\left(\mathbf{V}_{b}-\mathbf{u}\right) \cdot \mathbf{l}}{\left|\mathbf{V}_{b}-\mathbf{u}\right|}=\varepsilon\left(V_{b t}-u_{l}\right) \\
& t_{m}=\frac{\left|\mathbf{V}_{b}-\mathbf{u}\right|^{1 / m}}{\alpha^{1 / m}} \frac{\left(\mathbf{V}_{b}-\mathbf{u}\right) \cdot \mathbf{m}}{\left|\mathbf{V}_{b}-\mathbf{u}\right|}=\varepsilon\left(V_{b m}-u_{m}\right) \\
& t_{n}=\frac{\left(\mathbf{V}_{b}-\mathbf{u}\right) \cdot \mathbf{n}}{\beta}=\frac{1}{\beta}\left(V_{b n}-u_{n}\right) \\
& \varepsilon=\frac{\left|\mathbf{V}_{b}-\mathbf{u}\right|^{(1 / m)-1}}{\alpha^{1 / m}}=\frac{V_{S}^{(1 / m)-1}}{\alpha^{1 / m}},
\end{aligned}
$$

where $\mathbf{I}, \mathbf{m}$ are two tangential vectors and $\mathbf{n}$ is the outward unit normal vector on the wall surface and $\beta$ is the penalty parameter.

In most polymer processing operations, the constitutive equation of the polymer melts is modeled as generalized Newtonian fluids model with effective shear strain rate. As for viscosity, Cross model was used.

A weak form of the incompressible creeping flow problem is given by

$$
\begin{aligned}
& \int_{\Omega}(\nabla \cdot \sigma) \mathbf{v} d \mathbf{x}=0, \forall \mathbf{v} \in V \\
& \int_{\Omega}(\nabla \cdot \mathbf{u}) q d \mathbf{x}=0, \forall q \in Q
\end{aligned}
$$

where $V$ is the solution space of velocity, $Q$ is the solution space of pressure, $\mathbf{v}$ is an element of $V$ and $q$ is an element of $Q$. An element in $V$ must not only be square-integrable but its gradient must also be square-integrable, whereas only restriction on $Q$ is that it is a function space of squareintegrable functions.

By using the constitutive equation for generalized Newtonian fluids, divergence theorem, integral by part and taking boundary equations into account, Stokes flow problem can be stated in the following weak form:

$$
\begin{gathered}
\int_{\Omega} 2 \eta \mathrm{D}(\mathbf{u}): \mathbf{D}(\mathbf{v}) d \mathbf{x}-\int_{\Omega} p(\nabla \cdot \mathbf{v}) d \mathbf{x}=\int_{\Gamma} \mathbf{T} \cdot \mathbf{v} d \mathbf{x}, \forall \mathbf{v} \in V \\
\int_{\Omega}(\nabla \cdot \mathbf{u}) q d \mathbf{x} \approx 0, \forall q \in Q
\end{gathered}
$$

After introducing appropriate shape functions for velocity and pressure, substituting them into the above weak form results in nonlinear matrix equations as follows:

$$
\left[\begin{array}{cc}
{[K]+\left[K_{s}\right]} & -[G]^{T} \\
{[G]} & {[0]}
\end{array}\right]\left\{\begin{array}{c}
\{u\} \\
\{p\}
\end{array}\right\}=\left\{\begin{array}{c}
\left.\{f\}+\{\bar{F}\}_{s}\right\} \\
\{0\}
\end{array}\right\}
$$

where the subscript, $S$ represents a new added term which is related with the slip velocity.

One should account for the advancement of the melt front for the transient filling process as filling proceeds. At each time step, quasi-steady assumption is used. At the gate, velocity is prescribed throughout the filling. So it is possible to estimate the time to fill the gate node volumes. One should find frontal nodes that lie adjacent to gates and totally filled nodes, and therefore, filled elements that determine the computational domain to solve at each time step. A traction free boundary condition is imposed on the free surface. Only with the volume of the filled elements, one makes and solves the system of equations that corresponds to the current flow state. From the current velocity distribution, the volume flow rate and the time to fill each melt front node can be calculated. Finally, from the estimated filling time distribution at melt front nodes, some nodes to fill at a certain time increment can be chosen and the melt front is advanced. The above procedure is repeated to the end of filling.

\section{Applications of PIM Filling Analysis}

5.1 Quasi 3-Dimensional Filling Simulation for Electronic Package

The PIM filling analysis has been successfully applied to various mold geometries, including an ashtray shaped mold, mold for cutting tool die and alumina turbine rotor. The numerical results provide users with useful information - the melt front propagation patterns as a function of time, transient temperature field, transient pressure field, wall shear stress distribution, slip layer thickness over the mold surface (or slip velocity distribution over the mold surface. depending on the choice of the slip model), required clamping force, filling time, weld line locations, and so on. Such detailed information will help to improve the mold design and to optimize processing conditions.

In order to design the mold for electronic package, made by W-Cu mixture, quasi-3-dimensional PIM filling analysis program with slip layer model is used. Fig. 3 shows green, brown and final part of electronic package. Finite element 
mesh for electronic package simulation has 1797 linear triangular elements and 1004 nodes. Two gates are located on the edge of the frontal side. In Fig.4-6, filling pattern, temperature distribution and slip layer thickness distribution over the mold surface just at the end of filling is shown, respectively. From Fig.6, it is found that the maximum slip layer thickness occurs around gate due to the high shear rate. From this result, one can predict the region that has

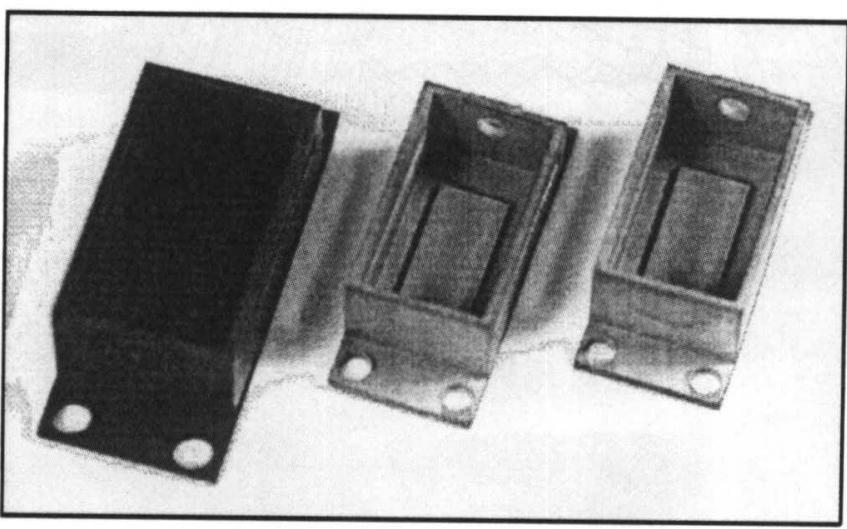

Fig.3 Green, brown and final part of electronic package.

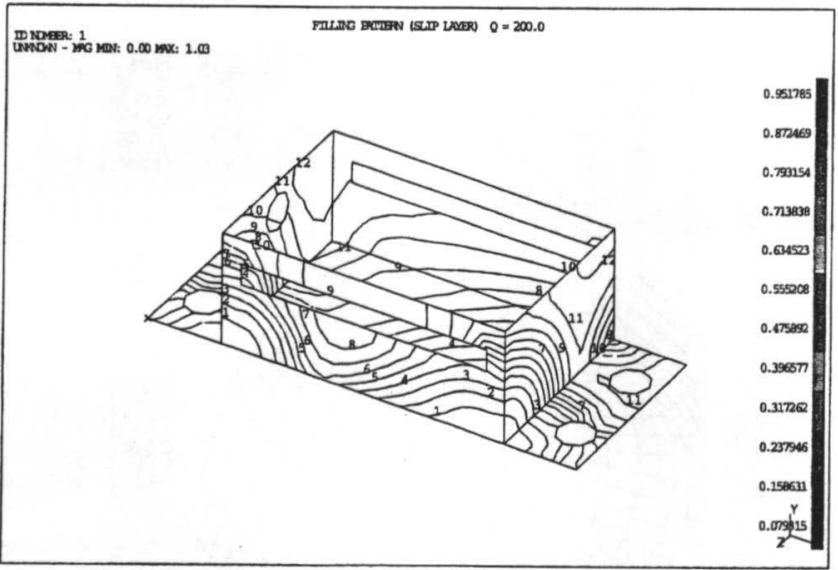

Fig.4 Finite element mesh for electronic package simulation.

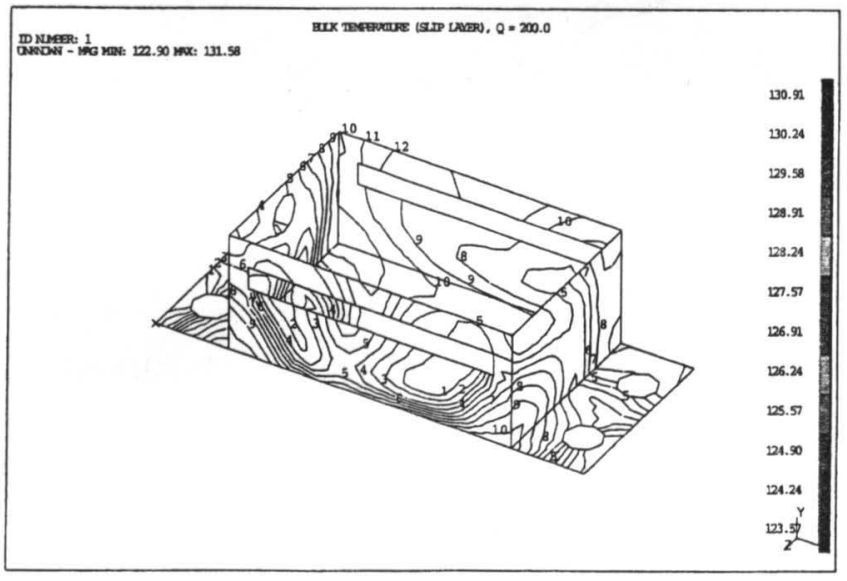

Fig.5 Filling patterns of electronic package. large possibility of binder separation.

5.2 Full 3-Dimensional Filling Simulation for P30 Insert

It should be noted that as part thickness increases, full 3dimensional flow effects (such as fountain flow) are significant. So it is impossible to apply the Hele-Shaw approximation such as P30 Insert mold shown in Fig.7. In this regard, full 3-dimensional analysis was executed for P30 Insert mold. Finite element mesh for P30 Insert with 2352 cubic elements and 3024 nodes was used. The gate is located on the center of the upper part in the backside. Fig. 8 shows the melt front advancement of P30 Insert simulation. The melt fronts advance symmetrically and meet in the lower part. It is matched well with experimental data as depicted in Fig.9.

\section{Acknowledgments}

The authors wish to thank the Korean Ministry of Science and Technology for their financial support through a research grant for the present work as a part of the Highly Advanced National Projects (Development of New Advanced-Materials for Information, Electronics and Energy Industry Division). A partial support via a research

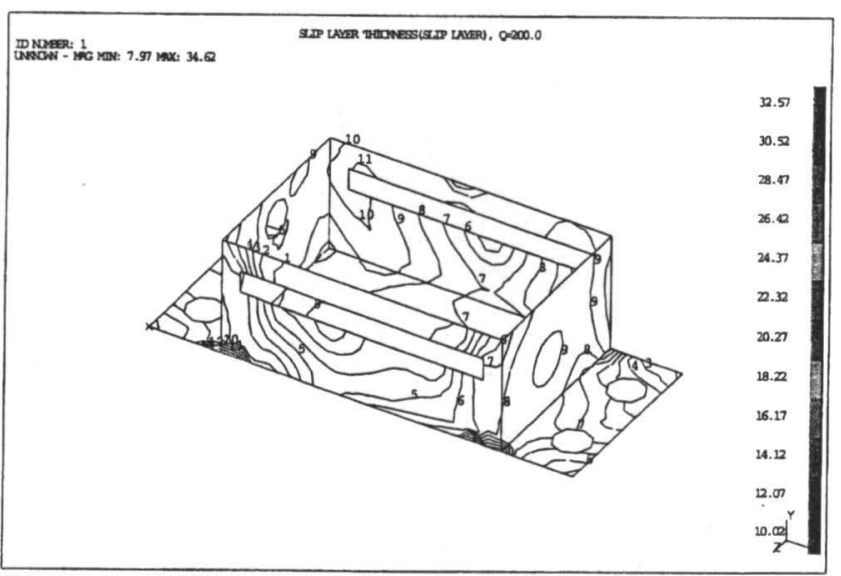

Fig.6 Temperature distribution at the end filling of electronic package.

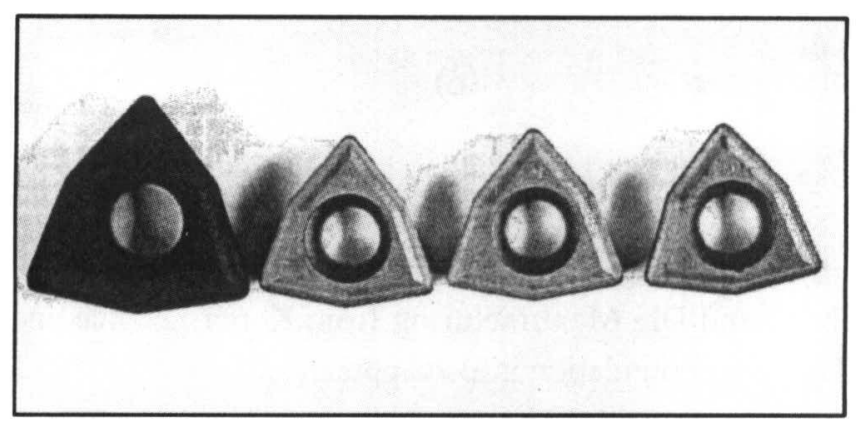

Fig.7 Slip layer thickness distribution over the mold surface of electronic package. 


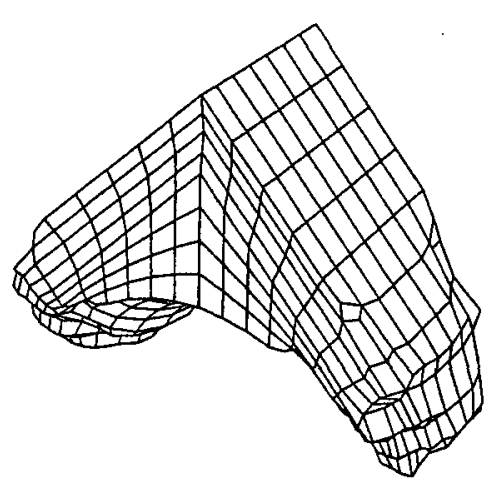

(a)

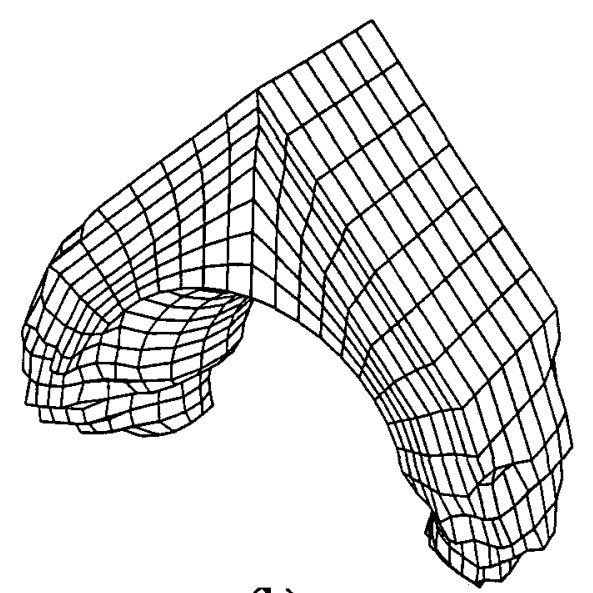

(b)

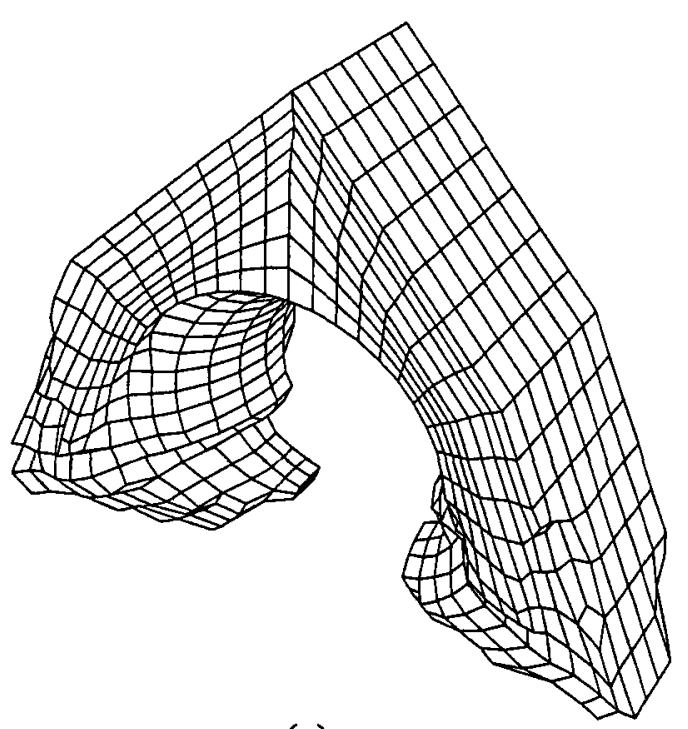

(c)

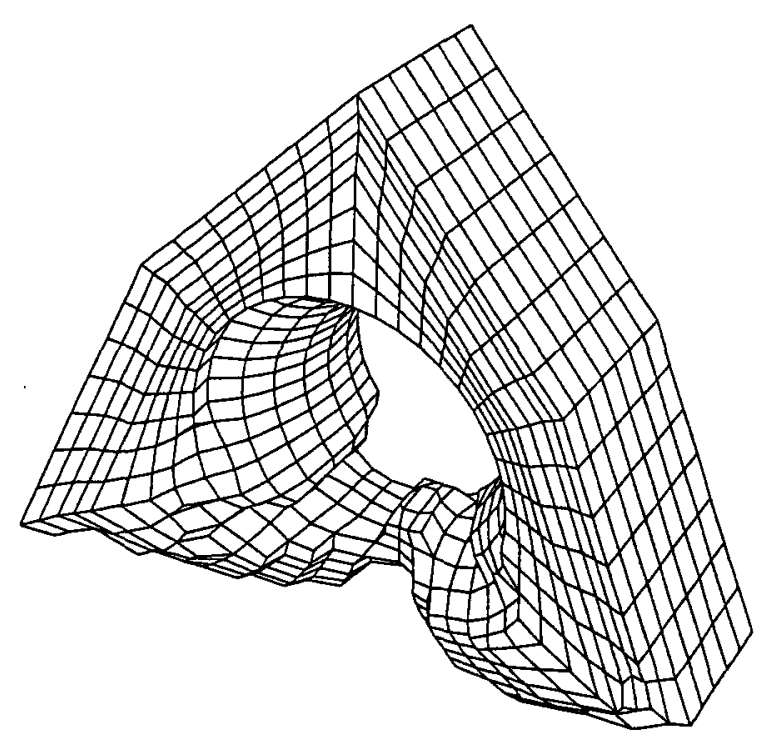

(d)

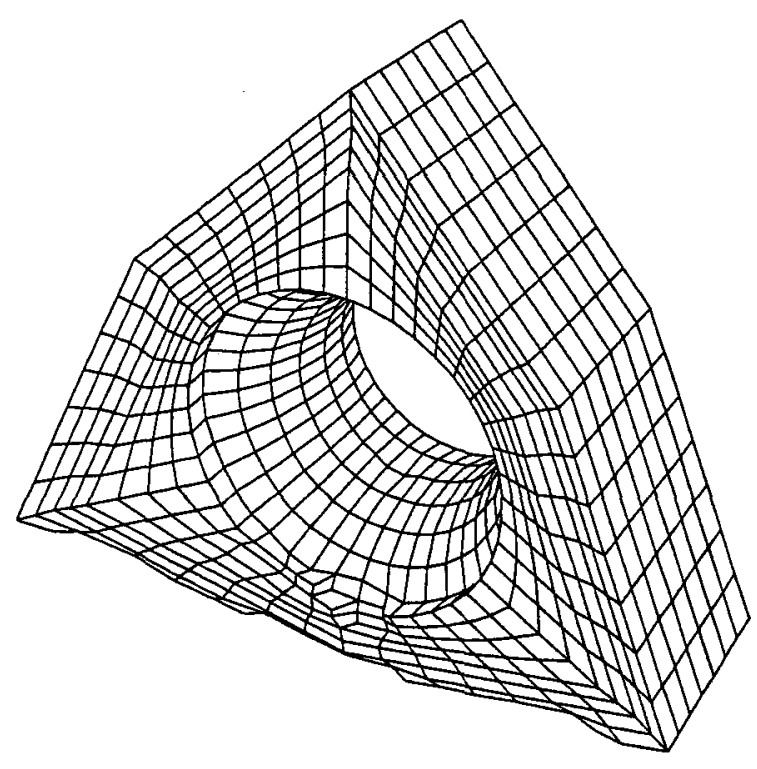

(e)

Fig.8 Green, brown and final part of P30 insert.

program through Engineering Research Center for NetShape and Die Manufacturing from Korea Science and Education Foundation is also appreciated.

\section{References}

1) R.M.German: Powder Injection Molding, Metal Powder
Industries Federation, Princeton, N.J., (1990) ch.1.

2) T.H.Kwon and J.B.Park: "Finite Element Analysis Modeling of Powder Injection Molding Filling Process Including Yield Stress and Slip Phenomena", Polym. Eng. Sci., 35(1995)741-753.

3) T.H.Kwon and S.Y.Ahn: "Slip Characterization of 


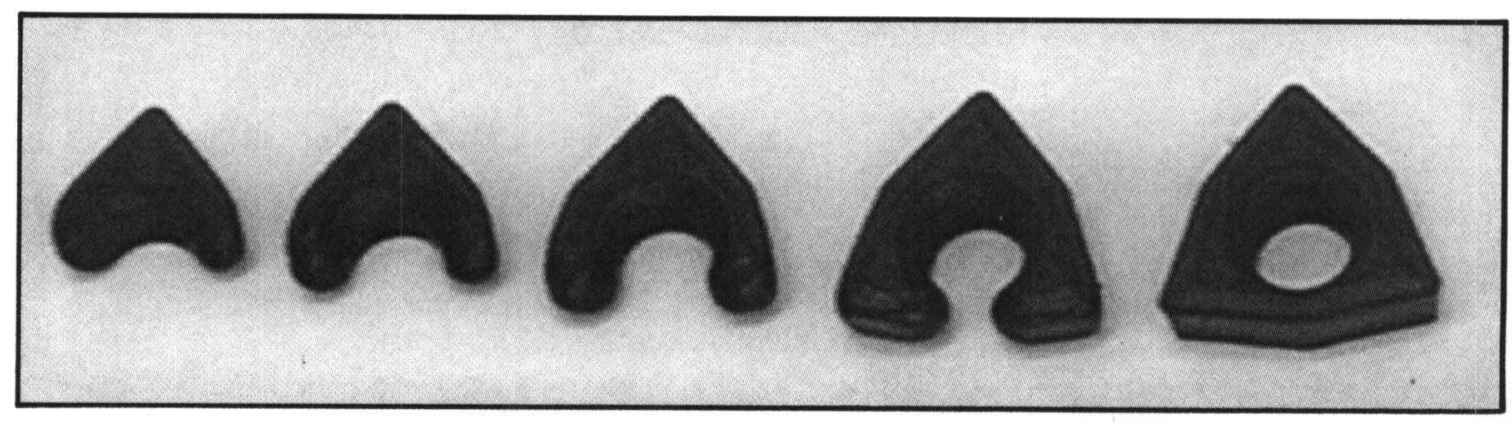

Fig.9 Full three-dimensional simulation of the melt front advancement.

Powder/Binder Mixtures and its Significance in the Filling Process Analysis of Powder Injection Molding", Powder Technol., 85(1995)45-55.

4) T.Q.Jiang, A.C.Young and A.B.Metzner: "The Rheological Characterization of HPG Gels: Measurement of Slip Velocities in Capillary Tubes", Rheol. Acta, 25(1986)397-404.

5) M.Mooney: "Explicity Formulas for Slip and Fluidity", J. Rheol., 2(1931)210.

6) U.Yilmazer and D.M.Kalyon: "Dilatancy of Concentrated Suspensions with Newtonian Matrices",
Polym. Compos., 12(1991)226-232.

7) D.M.Kalyon, P.Yaras, B.Aral and U.Yilmazer: "Rheological Behavior of a Concentrated Suspensions- A Solid Rocket Fuel Simulant", J. Rheol., 37(1993)35-53.

8) Z.Ji and A.D.Gotsis: "Numerical Study of Slip at the Walls in the Extruder", Intern. Polymer Processing, VII, 2(1992)132-139.

9) S.J.Kim and T.H.Kwon: "Development of Numerical Simulation Methods and Analysis of Extrusion Process of Particle-filled Plastic Materials Subject to Slip at the Wall", Powder Technology, 85(1995)227-239. 